\title{
¿n the Progressive Attenuation of Finescale Orography Contributions to the Vertical Coordinate Surfaces within a Terrain-Following Coordinate System
}

\author{
Syed Zahid Husain, Claude Girard, and Leo Separovic \\ Atmospheric Numerical Prediction Research Section, Meteorological Research Division, Environment and Climate Change \\ Canada, Dorval, Québec, Canada \\ André Plante And SHawn CoRvec \\ Canadian Meteorological Centre, Environment and Climate Change Canada, Dorval, Québec, Canada
}

(Manuscript received 11 March 2020, in final form 5 August 2020)

\begin{abstract}
A modified hybrid terrain-following vertical coordinate has recently been implemented within the Global Environmental Multiscale atmospheric model that introduces separately controlled height-dependent progressive decaying of the small- and large-scale orography contributions on the vertical coordinate surfaces. The new vertical coordinate allows for a faster decay of the finescale orography imprints on the coordinate surfaces with increasing height while relaxing the compression of the lowest model levels over complex terrain. A number of tests carried out-including experiments involving Environment and Climate Change Canada's operational regional and global deterministic prediction systems-demonstrate that the new vertical coordinate effectively eliminates terrain-induced spurious generation and amplification of upper-air vertical motion and kinetic energy without increasing the computational cost. Results also show potential improvements in precipitation over complex terrain.
\end{abstract}

KEYWORDS: Numerical weather prediction/forecasting; Semi-Lagrangian models; Vertical coordinates

\section{Introduction}

Terrain-following coordinates (TFCs)—based on pressure or height—have become the de facto choice for numerical weather prediction (NWP) models. The TFC approach maps the complex Earth surface in the physical domain to a flat surface in the computational domain. This simplifies the application of the lower boundary conditions and permits nearuniform vertical resolution for boundary layer representation across the entire computational domain irrespective of the local topography. Most NWP models utilize some form of a nonorthogonal TFC (Husain and Girard 2017; Wood et al. 2014). Although it is possible to construct orthogonal TFCs (e.g., Li et al. 2014), such coordinates are not common in atmospheric modeling.

Although the TFC approach has been widely adopted by the NWP community, it is known to suffer from two potential weaknesses that can adversely affect the evolution and representation of near-surface and upper-airflow. In the vicinity of the model surface with a TFC, sufficiently steep orography (usually slopes greater than $45^{\circ}$ ) can lead to significant error in the estimation of the horizontal gradients eventually leading to numerical instability (Vionnet et al. 2015; Husain et al. 2019). Such an instability could be addressed by improving the calculation of the horizontal gradients through modifications in the horizontal differencing stencils following the approach proposed by Mahrer (1984). The work by Zängl (2012) provides a

¿ Denotes content that is immediately available upon publication as open access.

Corresponding author: Syed Zahid Husain, syed.husain@canada.ca pertinent example of a numerical strategy inspired by Mahrer (1984) for improving model stability over steep slopes with a TFC.

The other significant limitation of the TFC approach is related to the imprint of orography in the vertical coordinate surfaces that can be significant even near the model top. The simplest form of TFC is a $\sigma$-type coordinate where the vertical coordinate surfaces are terrain-following near the surface and, depending on the choice of vertical coordinate, only becomes parallel to a constant-pressure or constant-height surface at the model top. This leads to spatial variations in the constant $\sigma$ surfaces in the upper troposphere and the stratosphere that, in the presence of strong horizontal wind, can induce widespread spurious circulations over complex terrain. In the context of two-dimensional flow over idealized mountains, Schär et al. (2002) have demonstrated that the $\sigma$-type TFC can lead to significant large-scale upper-air distortions in the vertically propagating gravity waves. For NWP, in addition to large-amplitude computational noise, such a coordinate can cause erroneous overprediction of upper-air turbulence (Park et al. 2019).

The limitation of the TFC concept with respect to upperairflow representation can be improved by hybridization of the TFC definition as proposed by Simmons and Burridge (1981). The vertical decay of orography imprints on the vertical coordinate surfaces is user-defined in a hybrid TFC. As a result, hybrid TFCs allow for a faster transition from terrainfollowing coordinate surfaces near the ground to constantpressure (or constant-height) surfaces with increasing height. A number of NWP models, including the operational GEM (Global Environmental Multiscale) model of Environment and Climate Change Canada (ECCC), are based on some 
form of a hybrid TFC (Girard et al. 2014). Furthermore, majority of the operational NWP models employ some form of semi-implicit (or iterative-implicit) time discretization that slows down the fastest moving waves along with semiLagrangian treatment for advection. Overall, such an approach allows large time-step lengths for attaining computational efficiency. As a result, these models permit Courant numbers that are often considerably larger than 1 , although the higherfrequency modes can suffer from incorrect resolution. In particular, with increasing model and orography resolution, large Courant numbers can lead to nonnegligible advection error at the upper-levels due to the combined effect of strong horizontal wind and the presence of finescale structures on the model levels. Regular hybrid TFCs are generally unable to address the underlying issue. The work by Schär et al. (2002) has convincingly illustrated this limitation of the hybrid TFC approach.

To improve upper-air predictions over complex orography with NWP models, Schär et al. (2002) proposed a simple solution that relies upon two separate vertical decay functions (instead of one) in the definition of the TFC for the contributions of the large-scale and finescale components of the orography. This modified TFC is referred to as a Smooth Level Vertical (SLEVE) coordinate. Within the SLEVE framework, the smaller-scales can be subjected to faster decay with height compared to the larger scales, and thereby reduce the numerical error associated with the finescale terrain imprints in the upper-air coordinate surfaces. For the two-dimensional mountain-wave problem presented by Schär et al. (2002), the SLEVE coordinate considerably reduces the large-scale distortions in the vertically propagating mountain waves. It is, however, important to note that the inconsistency in the calculation of departure height for the semi-Lagrangian model used by Schär et al. (2002) also contributed to the large-scale mountain-wave distortions (Girard et al. 2005; Husain and Girard 2017). Over the past years, several generalizations and extensions of the fundamental concept behind the SLEVE approach have been proposed that target finescale orography imprints in the vertical coordinate for increased progressive smoothing with height in order to improve upper-airflow representation (Leuenberger et al. 2010; Klemp 2011; Park et al. 2019).

Recently, a SLEVE-type TFC option was implemented within the GEM model's hybrid log-hydrostatic-pressurebased TFC (Husain and Girard 2017). The primary objective of the present work is to demonstrate the relative performance of a regular and a SLEVE-type hybrid coordinate in the context of an operational NWP model. In particular, the issue of error in the representation of upper-airflow with the regular hybrid coordinate-when subjected to finescale orographic forcing-is explored, and how a SLEVE-type modification to the coordinate definition can address the underlying issue in a computationally efficient way is documented. The paper is organized as follows. Section 2 provides the relevant background information on the GEM model as well as the regular and SLEVE-type hybrid vertical coordinate systems. Results pertaining to the principal objectives of the study using both limited-area and global test problems are presented in section 3. Finally, the conclusions derived from this research are summarized in section 4.

\section{Relevant background information}

\section{a. The GEM model}

The nonhydrostatic GEM model solves a system of equations originating from the compressible Euler equations. At present, the GEM model has options for two dynamical cores based on a pressure- (Girard et al. 2014) and a height-type (Husain et al. 2019) TFC, and these cores are referred to as GEM-P and GEM-H, respectively. However, all the GEMbased operational NWP systems are currently using the GEM$\mathrm{P}$ core, and therefore this paper will be entirely focused on the pressure-based vertical coordinate. Henceforth, GEM will actually refer to GEM-P in the remainder of this paper.

The spatial discretization in GEM utilizes the staggered Arakawa $\mathrm{C}$ grid in the horizontal and the Charney-Phillips staggering in the vertical. Time-stepping is based on a twotime-level Crank-Nicolson discretization along with semiLagrangian treatment for the advection terms. Extensive subgrid-scale parameterizations are implemented within the physics package of the model to account for the various physical processes like radiation, surface-atmosphere interactions, gravity wave drag, boundary layer mixing, condensation, cloud formation and precipitation (McTaggart-Cowan et al. 2019). During every model time step, the dynamical equations are first solved in the absence of any subgrid-scale physical forcing resulting in gridscale inviscid and adiabatic solution. The parameterized subgrid-scale physics tendencies are then applied to the dynamical core solution through gridpoint adjustments in the split mode to obtain the complete solution at the end of any time step. Further technical details on the GEM dynamical core are available in the existing literature (Girard et al. 2014; Husain and Girard 2017; Husain et al. 2019).

It is worth noting that the GEM dynamical core approaches both the regional and the global computational domains using limited-area model (LAM) latitude-longitude (lat-lon) grids. The global grid is based on the Yin-Yang system that combines two overlapping LAM grids (Qaddouri and Lee 2011).

\section{b. The regular hybrid coordinate}

The vertical coordinate in the operational GEM model follows the concept of the generalized hydrostatic-pressure-type hybrid coordinate proposed by Laprise (1992). It employs a loghydrostatic-pressure-type TFC (Girard et al. 2014) given by

$$
\ln \pi=\zeta+B s,
$$

where $\pi$ is the hydrostatic pressure, $\zeta$ defines the terrainfollowing vertical coordinate, $B$ is the vertical decay function that prescribes the rate of flattening of the vertical coordinate surfaces with elevation, and $s$ is to be determined from the boundary condition at the surface. The vertical coordinate $\zeta$ varies from $\zeta_{S}$ at the surface to $\zeta_{T}$ at the model top and the corresponding values of hydrostatic pressure are $\pi_{S}$ and $\pi_{T}$, respectively. 
The decay function $B$ in Eq. (1) is defined as

$$
B=\psi^{r} \equiv\left(\frac{\zeta-\zeta_{T}}{\zeta_{S}-\zeta_{T}}\right)^{r},
$$

where $r \geq 1$, and as a result $B$ varies from 1 at the surface to 0 at the model top. Further discussion on the exponent $r$ in Eq. (2) is provided later in this section. It is important to note that evaluating Eq. (1) at the model top gives $\zeta_{T}=\ln \pi_{T}$, whereas the value of the term $s$ can be obtained by evaluating the equation at the surface leading to

$$
s=\ln \pi_{S}-\zeta_{S},
$$

where $s$ is treated as one of the prognostic variables of the GEM dynamical core (Girard et al. 2014; Husain and Girard 2017).

The value of $\zeta$ at the surface is set to be equal to the log of a reference pressure value $p_{\text {ref }}=10^{3} \mathrm{hPa}$ (i.e., $\zeta_{S}=\ln p_{\text {ref }}$ ). At any given model vertical level, the value of $\zeta$ is then specified using the following relation:

$$
\zeta=\ln \left(\eta p_{\text {ref }}\right)
$$

where $0<\eta \leq 1$, with $\eta$ being equal to 1 at the model surface and decreases monotonically with elevation. The values of $\eta$ are user-defined, and they in turn determine the values of $\zeta$ for the constant $\zeta$ surfaces within the GEM model for a given model configuration.

The $B s$ term on the right-hand side of Eq. (1) adds the contribution of surface pressure, and thereby the model orography, on the constant $-\zeta$ surfaces. On the other hand, the exponent $r$ in the definition of $B$ imparts the hybrid aspect to this coordinate definition. For $r=1$, the coordinate behaves similar to a regular $\sigma$ coordinate, whereas for $r>1$, pressure variations within the constant $\zeta$ surfaces are rectified faster with altitude and, consequently, the coordinate in (1) transforms into a hybrid coordinate. A too large value of $r$ can lead to excessive squeezing of the coordinate surfaces over terrain slopes. This can result in large pressure-gradient error (Mahrer 1984), which can in turn induce numerical instability. It can be shown that $r \leq 3$ is generally a safe choice in this regard (see the appendix). To avoid too much squeezing of the vertical levels near the ground while allowing faster decay of the terrain imprints on the coordinate surfaces, the exponent $r$ is made a linear function of $\psi$ as

$$
r=r_{\max }-\left(r_{\text {max }}-r_{\text {min }}\right) \psi,
$$

having a minimum of $r_{\min }$ at $\psi=1$ (at the surface) and a maximum of $r_{\max }$ at $\psi=0$ (at the model top).

The variations of the decay function $B$ with respect to $\eta$ for different values of $r_{\min }$ and $r_{\max }$ are shown in Fig. 1 . It should be noted that the operational $15-\mathrm{km}$ global and $10-\mathrm{km}$ regional deterministic predictions systems of ECCC (known as the GDPS and RDPS, respectively) are currently using $r_{\min }=3$ and $r_{\max }=15$. This selection is based on the results from a series of extensive tests that were carried out in the past to ascertain the optimal values of $r_{\min }$ and $r_{\max }$ for regional and global NWP. As it can be seen from Fig. 1, for such a configuration $B$ remains nonzero until $\eta$ approaches 0.1 . Therefore,

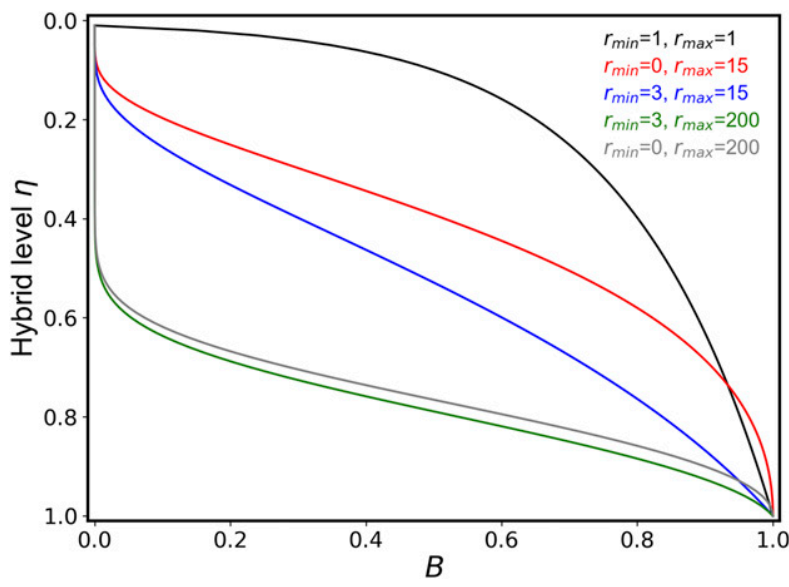

FIG. 1. Variations of the vertical decay function $B$ [see Eq. (2)] with respect to $\eta$ for different combinations of $r_{\min }$ and $r_{\max }$ values.

within the operational GDPS and RDPS, surface pressure imprints are permitted up until $100 \mathrm{hPa}$, above which the $\zeta$ surfaces essentially become parallel to the constant-pressure surfaces.

\section{c. The SLEVE-type hybrid coordinate}

The fundamental idea behind the SLEVE coordinate proposed by Schär et al. (2002) is to have two separate vertical decay functions for the large- and small-scale contributions of the terrain on the vertical coordinate surfaces. Following that principle, the vertical coordinate defined by Eq. (1) is modified as

$$
\ln \pi=\zeta+B_{L} s_{L}+B_{S}\left(s-s_{L}\right),
$$

to make it a SLEVE-type coordinate. In Eq. (6), the subscripts $L$ and $S$ refer to large and small scales, respectively. The largescale components of $s$ are evaluated by using the hydrostatic relation $(\partial \pi / \partial z)=-g \rho$, which in turn gives

$$
s_{L}=-g z_{L} /\left(R T_{L}\right)
$$

where the equation of state for ideal gas, $\rho=\pi /(R T)$, has been used. In Eq. (7), $z_{L}$ defines the large-scale orography, $T_{L}$ is the associated temperature, and $R$ is the universal gas constant. The appropriate choice of the cutoff length scale in terms of the model grid length is to be determined through numerical experiments in section 3 .

Both of the decay functions in Eq. (6) follow the same definition presented in Eq. (2). However, instead of one set of minimum and maximum values of $r$, the SLEVE framework utilizes two sets of these values (i.e., $r_{L \text {, min }}$ and $r_{L \text {, max }}$ for the large scales, and $r_{S \text {, min }}$ and $r_{S \text {, max }}$ for the small scales). The basic idea is that with $r_{S \text {, min }}>r_{L, \max }$, the SLEVE-type coordinate permits a faster rectification of the small-scale terrain imprints on the vertical coordinate surfaces with increasing height. This definition allows for a scale-selective squeezing of the coordinate surfaces over the mountains. It is also evident that with $r_{L, \text { min }}=r_{S, \text { min }}$ and $r_{L, \text { max }}=r_{S, \text { max }}$ the SLEVEtype coordinate reduces to the regular hybrid coordinate defined by Eq. (1). 


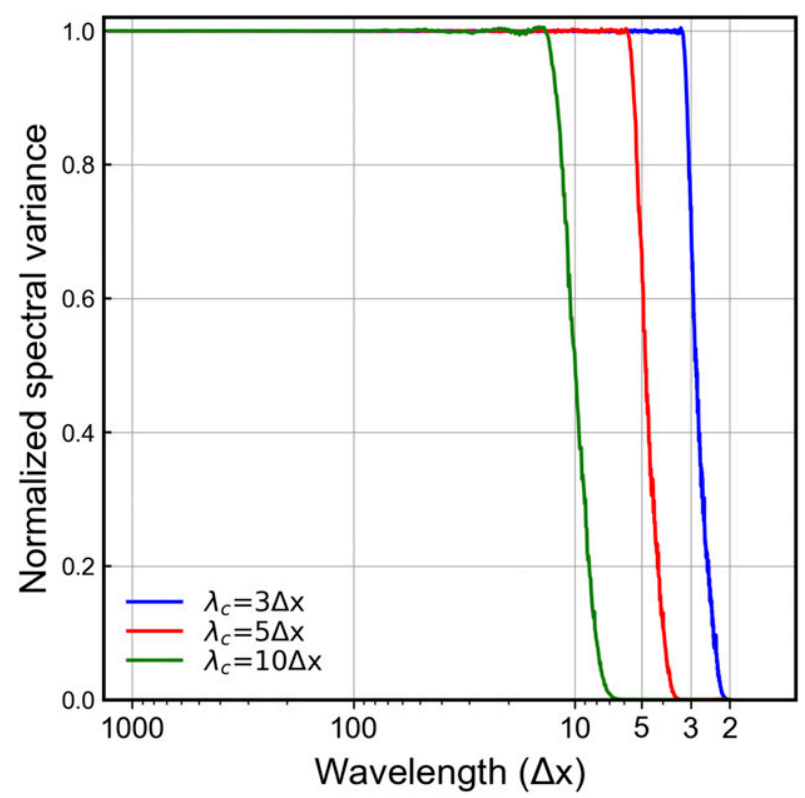

FIG. 2. Spectral response of the orography filter used in GEM with respect to different values of cutoff wavelength $\lambda_{c}$.

Henceforth, in this paper, the regular and SLEVE-type hybrid TFCs in GEM are referred to as the RHC (regular hybrid coordinate) and SHC (SLEVE-type hybrid coordinate), respectively. Furthermore, for convenience of notation, instead of providing $r_{\min }$ and $r_{\max }$ values for the large and small scales separately, from this point onward, the exponent $r$ of the vertical decay function $B$ is expressed as $r=\left(r_{\min }, r_{\max }\right)$ for RHC and $r=\left(r_{L, \text { min }}, r_{L, \text { max }}, r_{S, \text { min }}, r_{S, \text { max }}\right)$ for SHC.

A major concern with any hybrid TFC is the potential for excessive squeezing of the lowest model levels around the mountain tops over complex terrain. However, the additional degree of freedom allowed by the SHC approach-in terms of the separation of scales along with the corresponding distinct vertical decay functions-allows to have a faster decaying of the small-scale orography imprints with height without causing excessive squeezing of the vertical levels near the mountain tops. In other words, the benefit that can be obtained with the SHC approach does not come at the expense of potential numerical instability due to additional compression of the lowest model levels.

\section{Experimental setup and results}

This section presents the comparative performance of the RHC and SHC in the context of test problems involving both limited-area and global computational domains.

The resolved-scale orography for the different experiments presented in this paper are obtained with the application of a low-pass filter. The filter works by applying a smoothing operator on the orography field in the gridpoint space (Zadra 2018). Output of this filter depends on the associated smallscale cutoff parameter $\lambda_{c}$, which is defined by the user. For an orography field with a spatial resolution of $\Delta x$, a cutoff value of $\lambda_{c}=n \Delta x$ results in a filtered field where only $50 \%$ of the
TABLE 1. List of various experiments and the associated configurations. The exponent $r$ within the vertical decay function $B$ can have two values $\left(r_{\min }, r_{\max }\right)$ with RHC and four values $\left(r_{L, \min }\right.$, $\left.r_{L, \text { max }}, r_{S, \text { min }}, r_{S, \text { max }}\right)$ with SHC.

\begin{tabular}{lllc}
\hline \hline Expt name & $\lambda_{c}$ & \multicolumn{1}{c}{$r$} & $\Delta t(\mathrm{~s})$ \\
\hline RHC-01 & $3 \Delta x$ & $(3,15)$ & 300 \\
RHC-02 & $5 \Delta x$ & $(3,15)$ & 300 \\
RHC-03 & $3 \Delta x$ & $(3,15)$ & 100 \\
SHC-01 & $3 \Delta x$ & $(3,15,0,200)$ & 300 \\
SHC-02 & $3 \Delta x$ & $(0,10,0,200)$ & 300 \\
\hline
\end{tabular}

variance at $n \Delta x$ wavelengths are retained. The spectral response of this filter for the different cutoff length scales is shown in Fig. 2. Sharpness of the filter can also be controlled. For the sharpest configuration of the filter, with $\lambda_{c}=n \Delta x$ all wavelengths smaller than $(n-1) \Delta x$ are completely removed whereas wavelengths larger than $(n+1) \Delta x$ are fully retained (see Fig. 2 for $\lambda_{c}$ values of $3 \Delta x$ and $5 \Delta x$ ). It is important to note that, unless stated otherwise explicitly, the large-scale orography required for the different SHC experiments are obtained using the same low-pass filter with the small-scale cutoff wavelength set to $\lambda_{s}=10 \Delta x$. This leads to a large-scale orography field where variance of $13 \Delta x$ wavelength is fully retained, while length scales smaller than $7 \Delta x$ are completely eliminated (see Fig. 2 for $\lambda_{c}=10 \Delta x$ ). The small-scale orography field for SHC is then obtained by simply subtracting the large scales from the complete orography. A list of the principal configurations of the different numerical experiments pertaining to this study are presented in Table 1.

\section{a. The LAM test problem}

A small LAM test problem is devised in order to evaluate the impact of the different types of vertical coordinate configurations in a computationally efficient manner. The domain for this test case covers a small section over the Canadian Rockies while its grid points are collocated with ECCC's operational $10-\mathrm{km}$ resolution RDPS grid. However, unlike the orography in the operational RDPS that is obtained with $\lambda_{c}=$ $5 \Delta x$ (RHC-02 configuration in Table 1), the orography for this test case is based on $\lambda_{c}=3 \Delta x$ (RHC-01 configuration). This test case therefore permits more resolved fine scales in the orography and thereby allows to better highlight the upper-air issues related to TFCs. Moreover, it is important to note that the orography in ECCC's operational global system (GDPS) is obtained with $\lambda_{c}=3 \Delta x$. As a result, the conclusions derived by analyzing this small-domain LAM test problem can be validated through further testing with the GDPS (to be presented in a later subsection) and comparing the outcome with the operational GDPS.

The LAM test case is initialized at 0000 UTC 16 January 2016. The orography over the LAM domain for this case along with the wind vectors at $250 \mathrm{hPa}$ after $24 \mathrm{~h}$ of model integration are shown in Fig. 3a. The results show strong horizontal westsouthwesterly wind north of the Rockies with the maximum wind speed approaching approximately $75 \mathrm{~m} \mathrm{~s}^{-1}$. This is quite representative of the typical winter conditions for this region. 
a)

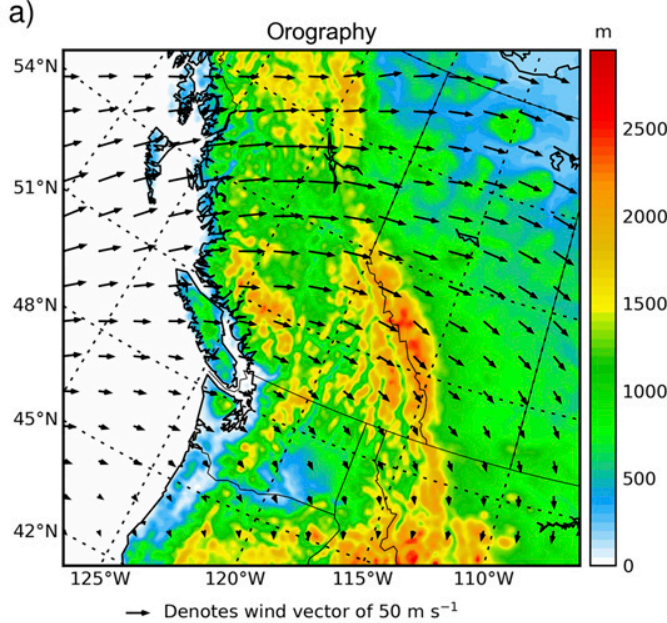

C) Vertical levels $\left(\lambda_{c}=3 \Delta x ; r=3,15\right)$

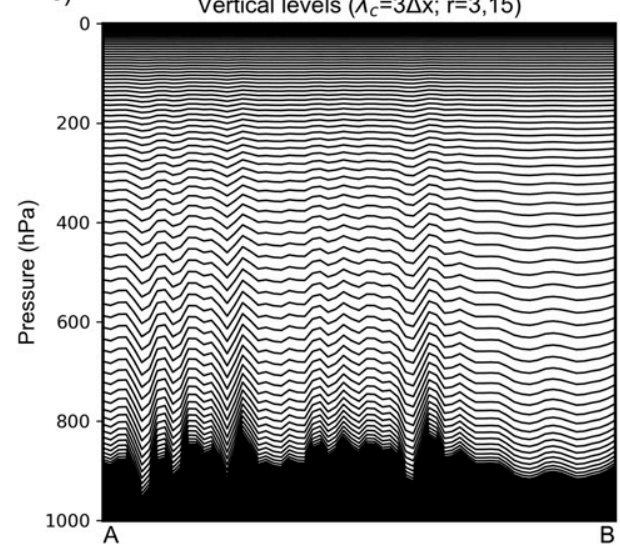

b)
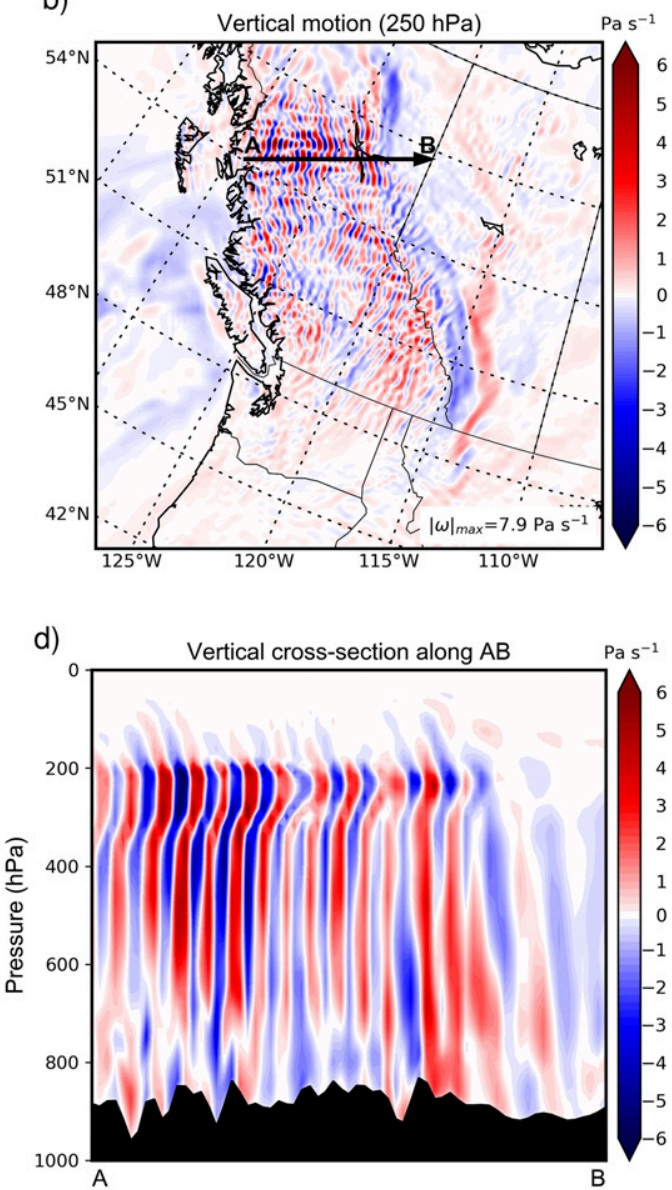

FIG. 3. (a) Orography over the computational domain of the LAM test case for the RHC-01 configuration (see Table $1 ; \lambda_{c}=3 \Delta x$ in the filter). The arrows represent the horizontal wind vector after $24 \mathrm{~h}$ of integration (initialized at 0000 UTC 16 Jan 2017). (b) The corresponding vertical motion $\omega$ at $250 \mathrm{hPa}$. The line AB is identified in this figure for later use in presenting the vertical cross section of $\omega$ obtained with different numerical experiments. The coordinates of points $\mathrm{A}$ and $\mathrm{B}$ are $54.046^{\circ} \mathrm{N}, 129.278^{\circ} \mathrm{W}$ and $56.743^{\circ} \mathrm{N}, 119.278^{\circ} \mathrm{W}$, respectively. (c) Distribution of the model vertical levels along $\mathrm{AB}$ for this configuration. (d) Vertical cross section of $\omega$ along the line $\mathrm{AB}$ valid at the same time as in (a).

To evaluate the different vertical coordinate configurations it is useful to inspect the vertical motion field $\omega$, defined as

$$
\omega=\frac{d p}{d t},
$$

where $p$ is the pressure and $d / d t$ represents the material derivative. The model prediction for $\omega$ with the RHC-01 configuration after $24 \mathrm{~h}$, presented in Fig. 3b, reveals substantial small-scale noise with structures primarily of $3-4 \Delta x$ wavelengths over a large part of British Columbia (BC). The maximum value of $|\omega|$ at this level is predicted to be $7.9 \mathrm{~Pa} \mathrm{~s}^{-1}$.

To better understand the noise in $\omega$, it will be useful to look at its vertical cross section around a region with strong horizontal wind and large-amplitude vertical motion. This leads to the selection of the line AB shown in Fig. 3b. The distribution of the model vertical levels along $\mathrm{AB}$ is presented in Fig. 3c, whereas Fig. $3 d$ provides the vertical cross section of $\omega$ along the same line. As RHC-01 is based on $\lambda_{c}=3 \Delta x$, the vertical cross section shows significant finescale orography. With $r=$ $(3,15)$ in RHC-01, although the large-scale orography imprints are largely smoothed out at around $200 \mathrm{hPa}$, the finescale orography imprints on the model vertical levels (and by extension on the model vertical coordinate surfaces) persist. With the horizontal wind speed being quite large, any considerable finescale pressure fluctuations along the model vertical coordinate surfaces can lead to significant local error in advection calculations, and thereby lead to spurious generation and amplification of $\omega$ at the upper levels (around $250 \mathrm{hPa}$ ).

Any issue pertaining to the presence of finescale orography imprints in the model surfaces around and above the tropopause can be simply addressed by using an orography obtained with $\lambda_{c}>3 \Delta x$ in the filter. For example, the operational RDPS that utilizes $\lambda_{c}=5 \Delta x$ (RHC-02 configuration in Table 1 ), and thus have a significantly smoother orography to begin with. 
a)

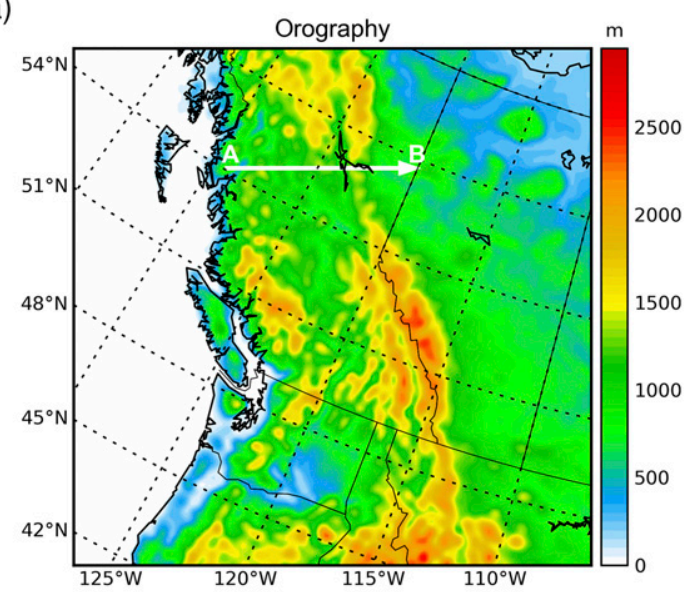

b)

d)

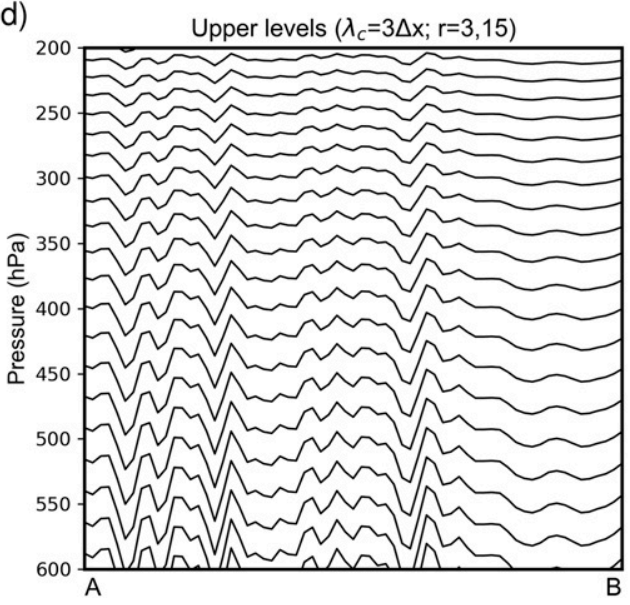

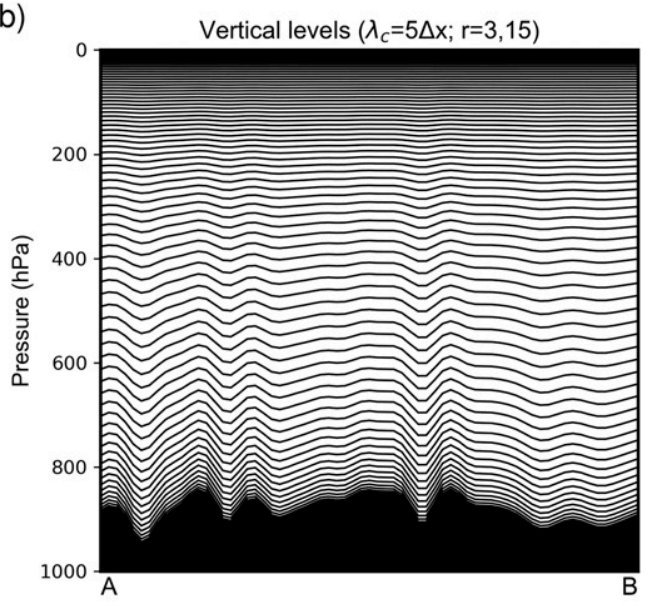

c)

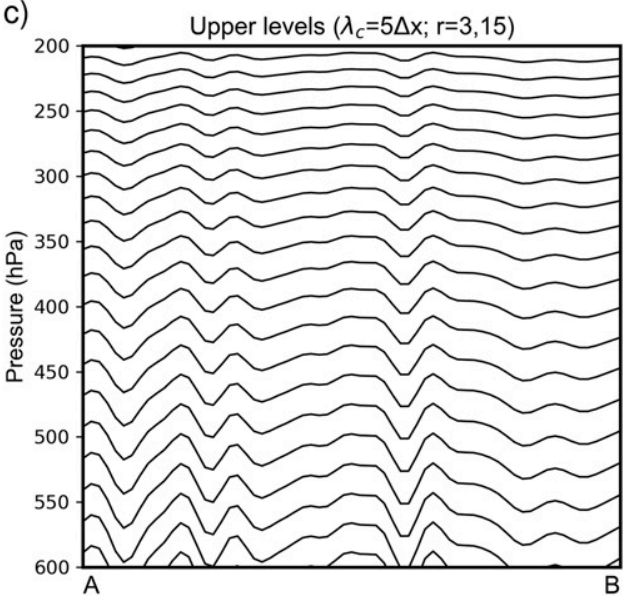

\section{.}


a)

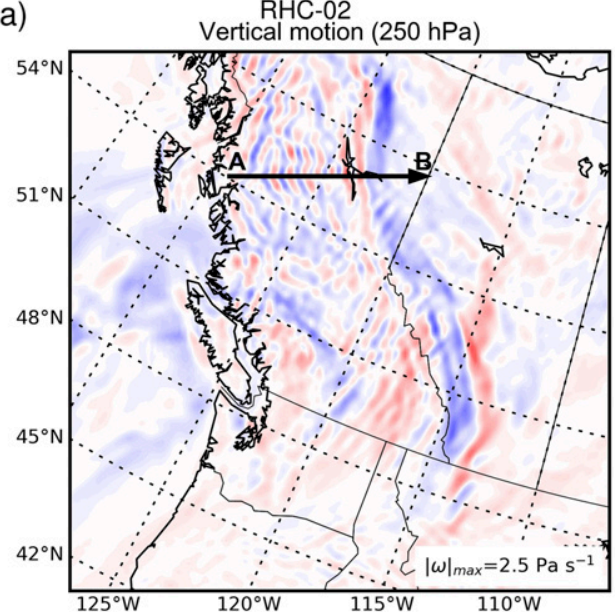

c)

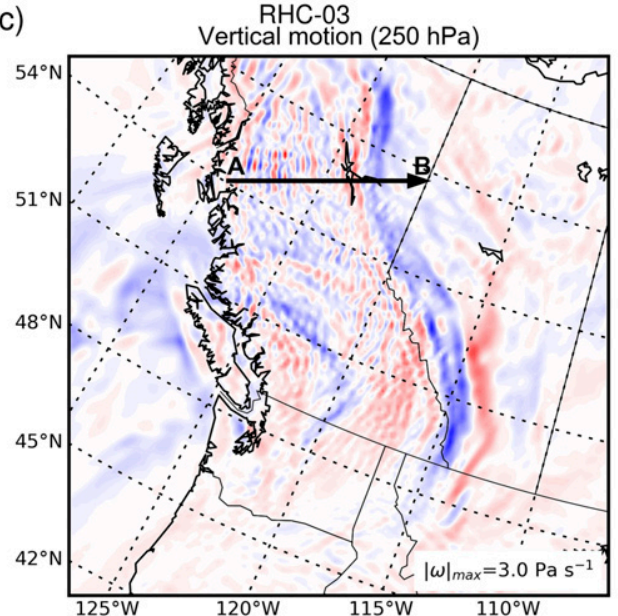

b)

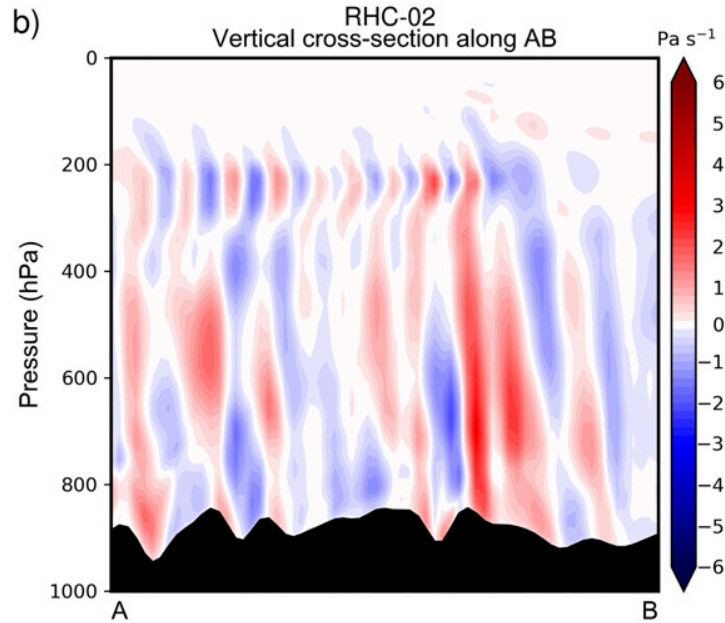

d)

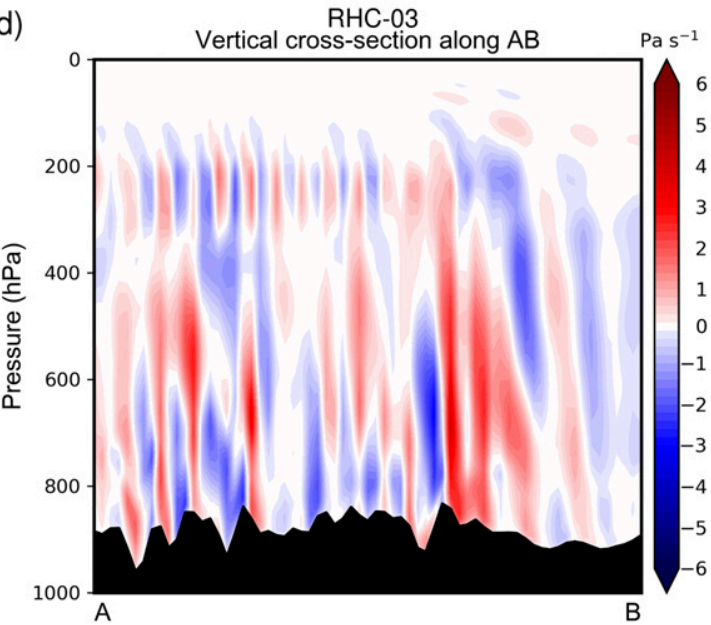

FIG. 5. Vertical motion $\omega$ at $250 \mathrm{hPa}$ after $24 \mathrm{~h}$ with: (a) RHC- 02 and (c) and RHC- 03 configurations. Vertical cross section of $\omega$ along AB with (b) RHC-02 and (d) RHC-03 configurations. All other conditions as in Fig. 3.

shown in Figs. 6a and 6b. For kinetic energy, the spectra corresponding to the three configurations almost coincide for wavelengths larger than $5 \Delta x$. Below $5 \Delta x$, the RHC-01 configuration shows flattening of the spectral slope within the 3-5 $\Delta x$ range implying numerical amplification of variance at these length scales. The issue is more evident in the spectra of $\omega$ where the RHC-01 configuration clearly shows a spurious spike in variance for the same length scales. Reduction of timestep length (RHC-03 configuration) shows a substantial reduction in the variance at these length scales although some signature of the spurious amplification is still visible, particularly in $\omega$. In fact, even with the RHC-02 configuration where the actual orography is considerably smoother, careful inspection of the $\omega$ spectra reveals some signs of increase in variance around the cutoff length scale of $5 \Delta x$. As the spectra of the orography at these scales do not suffer from any increase in variance at the cutoff length scales (not shown), these bumps in the $\omega$ spectra are apparently also partly related to the accuracy of the underlying numerics employed in the model.

Overall, the results presented in Figs. 5 and 6 show that filtering the model orography and reducing the model time-step length provide means for controlling the spurious TFC-related increase in variance of upper-air kinetic energy and vertical motion as well as other model-predicted meteorological fields of interest. However, at present the focus at the different operational NWP centers is to improve the representation of orography details (Elvidge et al. 2019), which makes any aggressive filtering of the orography unacceptable. Furthermore, for operational NWP models the emphasis is always on producing forecasts in a computationally efficient way. Reducing the time-step length is therefore an even more problematic proposition, particularly when a direct solver - that has a fixed cost per time-step as in GEM-is used. Development and implementation of SHC in GEM is therefore motivated by the limitations of these alternate fixes.

The model vertical levels along AB (see Fig. 4a) for two different SHC configurations are shown in Fig. 7. The first configuration, SHC-01, is based on $r=(3,15,0,200)$. Thus it maintains the same decaying with height for the large scales while rapid decaying is applied to the small scales. The SHC-02 configuration on the other hand utilizes $r=(0,10,0,200)$, and thus relaxes the large-scale decaying with height while 
a)

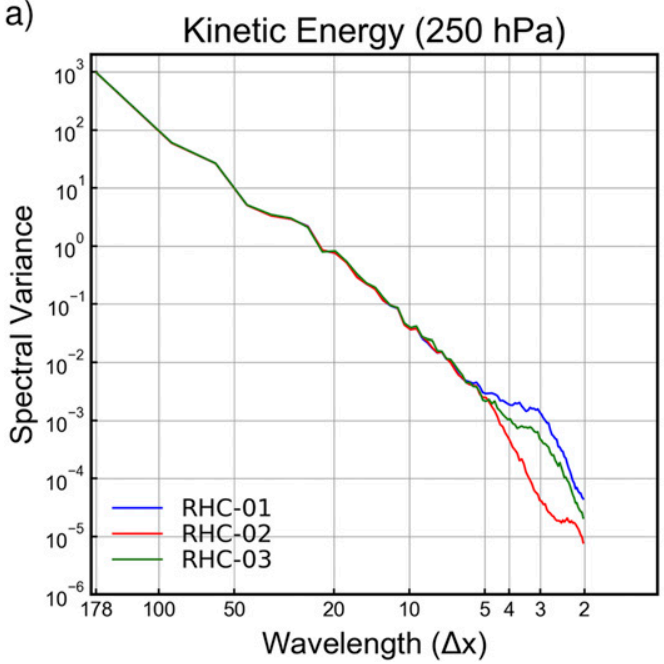

b)

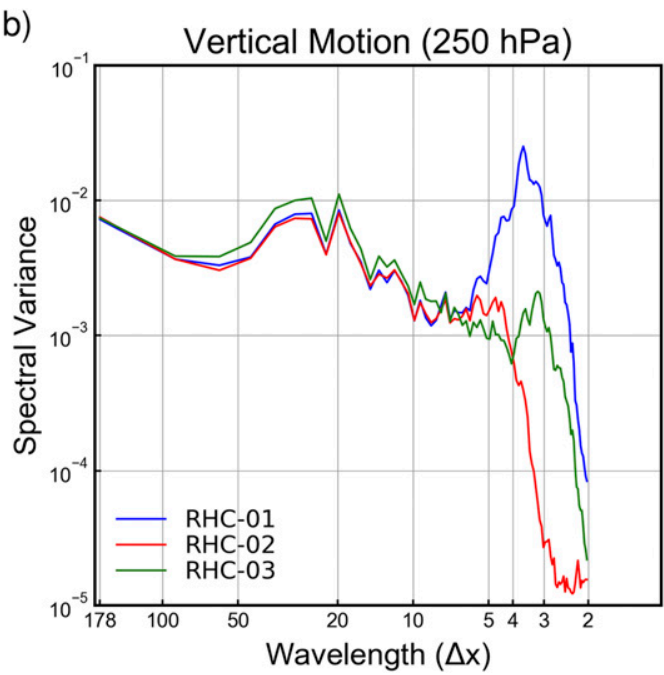

FIG. 6. Spectral variance as a function of wavelengths in terms of grid spacing $(\Delta x=10 \mathrm{~km})$ in (a) kinetic energy and (b) vertical motion at $250 \mathrm{hPa}$ after $24 \mathrm{~h}$ of simulation for three different vertical coordinate configurations: RHC-01 (blue), RHC-02 (red), and RHC-03(green).

maintaining the same rate of decay for the smallest length scales with height as in SHC-01. Above $500 \mathrm{hPa}$, as can be seen in Fig. 7, although SHC-02 shows more large-scale variance in the model levels, it leads to very similar variance in the small scales. In fact, as far as the smallest scales are concerned, both SHC-01 and SHC-02 are substantially smoother compared to the RHC-01 configuration (see Fig. 4d). Vertical motion at $250 \mathrm{hPa}$ and its vertical cross section along $\mathrm{AB}$ for the two SHC configurations are presented in Fig. 8. It is evident that both of the SHC configurations lead to similar degree of elimination of the spurious upper-level amplification of vertical motion. The maximum amplitude of the vertical motion at $250 \mathrm{hPa}$ is also reduced to approximately $2.5 \mathrm{~Pa} \mathrm{~s}^{-1}$ by both configurations. Although, the maximum vertical motion between RHC-03 and the two SHC configurations are comparable, there are nevertheless considerable differences in the $\omega$ distribution at around $250 \mathrm{hPa}$. These differences are due to the fact that none of these TFC configurations resulted in a temporal convergence with respect to the advection error. In fact, further tests have revealed that convergence is only achieved when the time-step length is reduced to $\Delta t=50 \mathrm{~s}$ and $\Delta t=150 \mathrm{~s}$ with the RHC and SHC configurations, respectively (not shown). This again underscores a significant computational efficiency advantage for the SHC approach for obtaining similar accuracy. However, in the context of operational NWP systems, the goal of this study is not to eliminate the error, rather to reduce the error-in terms of the maximum amplitude of $\omega$ - to an acceptable level, as found with the filtered orography experiment (i.e., the RHC-02 configuration). a)

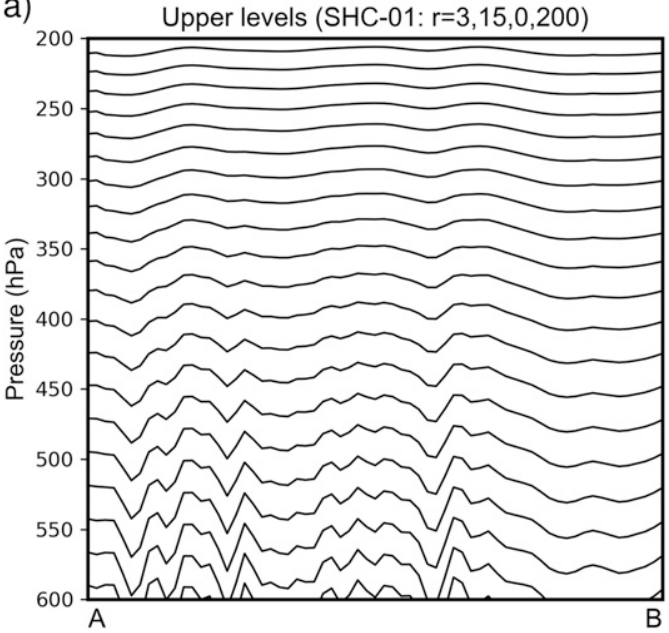

b)

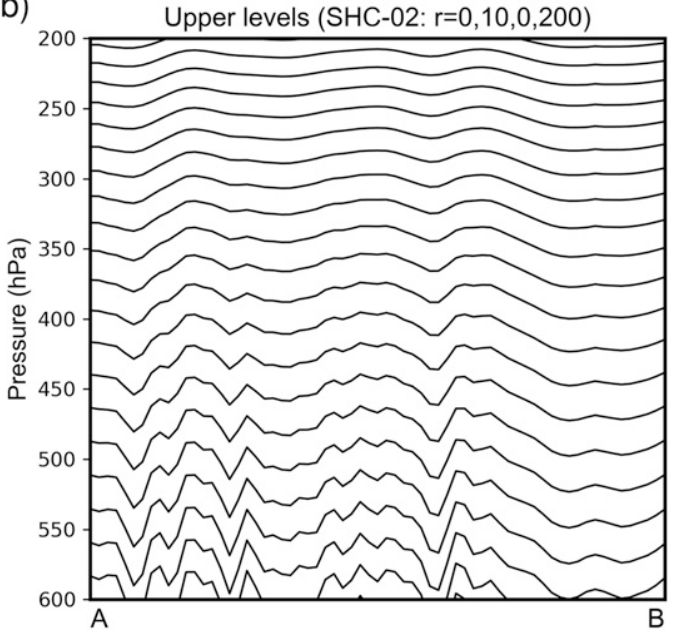

FIG. 7. Upper-air vertical level structures along AB for two SLEVE-type hybrid coordinate configurations:

(a) SHC-01 and (b) SHC-02. 
a)

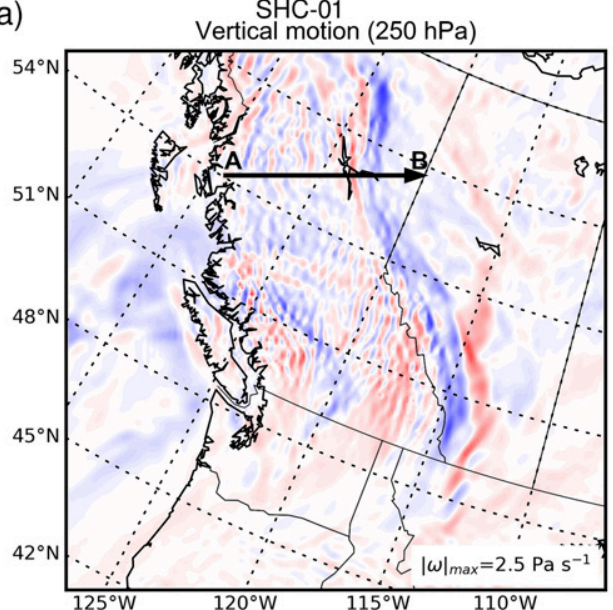

c)

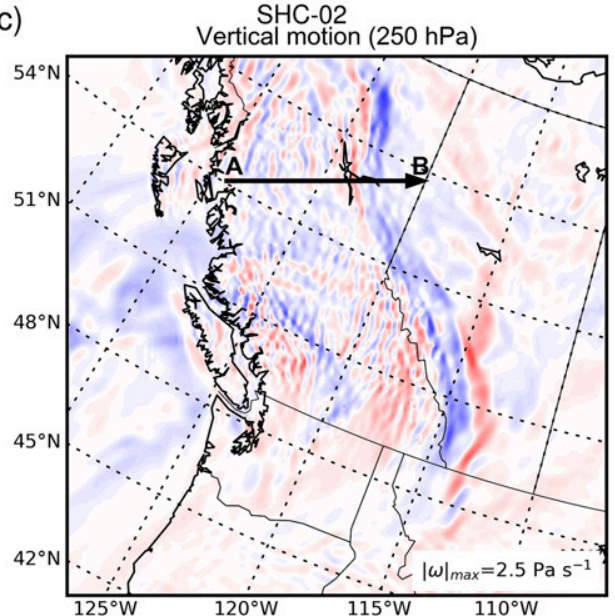

b)

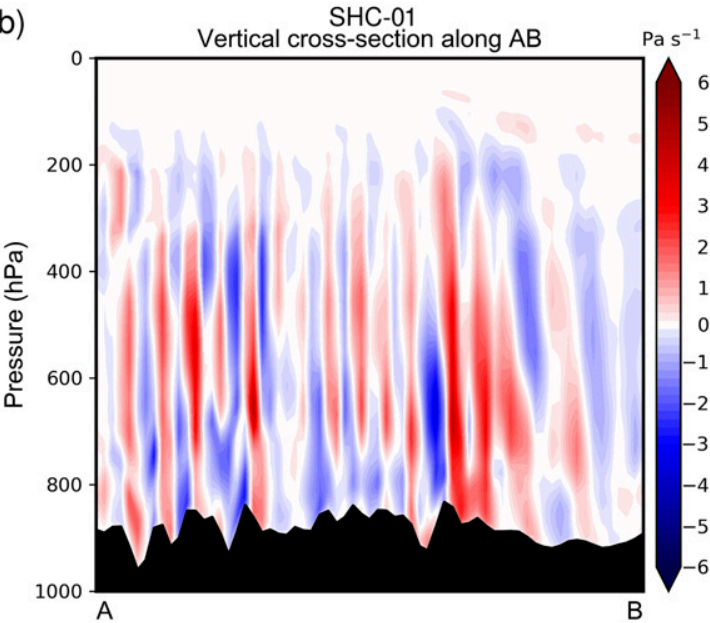

d)

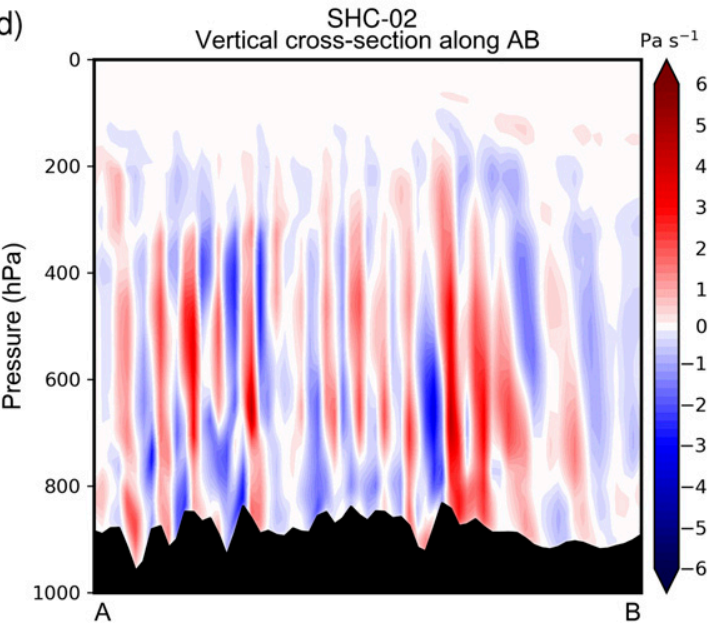

FIG. 8. As in Fig. 5, but for two different configurations of SLEVE-type hybrid coordinate: (top) SHC-01 ( $r=3$, $15,0,200)$ and (bottom) SHC-02 $(r=0,10,0,200)$. The small scales for both cases are obtained with $\lambda_{s}=10 \Delta x$ in the orography filter.

The substantial improvements in small-scale variance is further demonstrated with the help of kinetic energy and vertical motion spectra in Fig. 9. The finescale variance around the small-scale cutoff wavelength $\lambda_{c}$ is reduced by more than an order of magnitude in vertical motion and around an order of magnitude for kinetic energy. These results clearly demonstrate the benefit of a SLEVE-type approach particularly when the model grid resolution is continuously being increased within the different operational NWP systems while also utilizing sharp orography filters as the one in GEM.

As has been mentioned earlier, the large-scale orography for SHC, for the different experiments presented so far, is obtained with a small-scale cutoff in the filter set to $\lambda_{s}=10 \Delta x$. The sensitivity of the underlying numerical issue to the smallscale cutoff wavelength $\lambda_{s}$ for the SHC-02 configuration is shown in Fig. 10. The figure reveals that retaining scales as large as $15 \Delta x$ in the small-scale orography can lead to considerable spurious increase in variance at the finest scales $(\leq 4 \Delta x)$ in both kinetic energy and $\omega$. This implies that the benefits of SHC may diminish as the range of length scales within the small-scale orography becomes too large. On the other hand, when small-scale cutoff is too small (e.g., $6 \Delta x$ or lower), it reduces the effectiveness of SHC for scales slightly larger than the cutoff length scale, and as a result, leads to spurious increase in variance within the 6-9 $6 x$ range for the vertical motion. The tests carried out for this study therefore show that the optimal range for small-scale cutoff wavelength with SHC

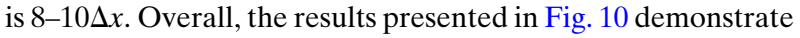
that the choice of $\lambda_{s}$ cannot be arbitrary and is likely related to the effective model resolution that is dictated by the smallest length scales in terms of the model grid resolution that a model is capable of resolving with acceptable accuracy. Various studies in the past have established the effective resolution for NWP models in general, and advection in particular, to be in

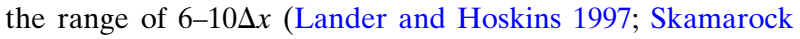
2004). This is consistent with the optimal cutoff length scale for SHC obtained in this study. In general, it is always prudent to avoid imposing stationary forcing at scales below a model's effective resolution. Furthermore, it is worth noting that the effective resolution, and thereby the optimal cutoff wavelength, 
a)

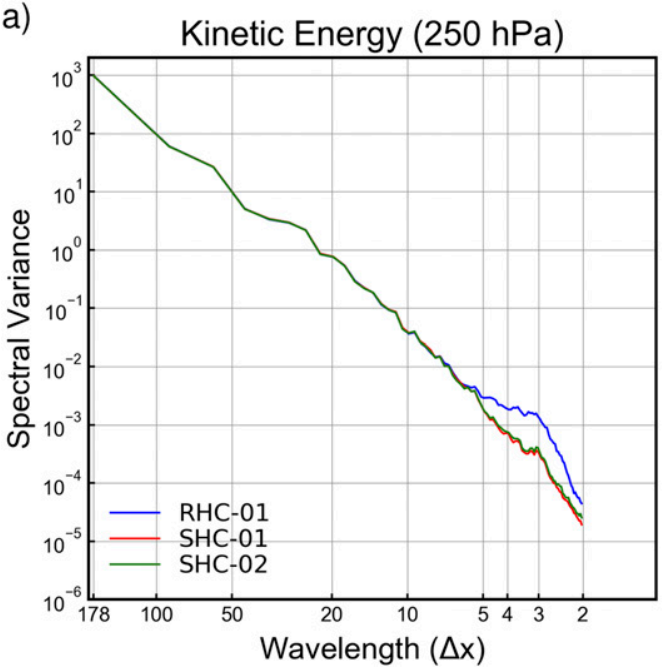

b)

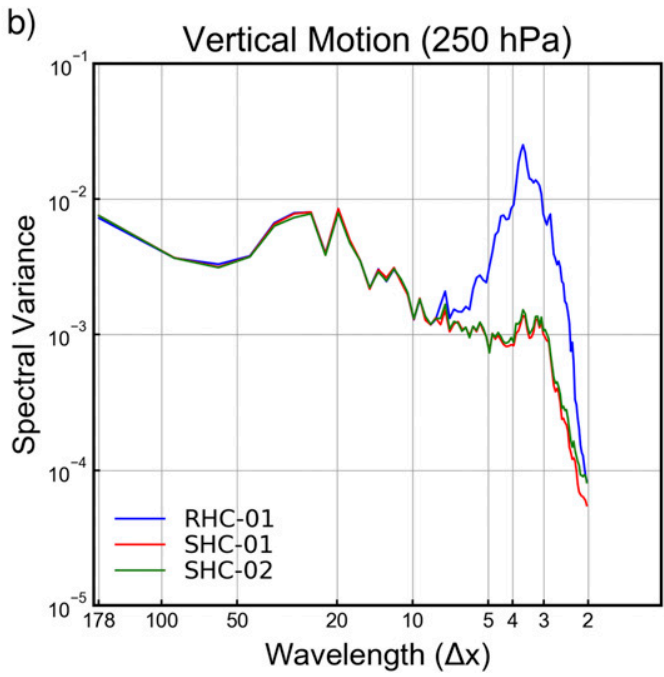

FIG. 9. Spectral variance as a function of wavelengths in terms of grid spacing $(\Delta x=10 \mathrm{~km})$ in (a) kinetic energy and (b) vertical motion at $250 \mathrm{hPa}$ after $24 \mathrm{~h}$ of simulation for three vertical coordinate configurations of regular and SLEVE-type: RHC-01 (blue), SHC-01 (red), and SHC-02 (green).

may depend on the numerical details of an NWP model. Therefore, if the SHC approach proposed in this study is to be adopted in any other NWP model, special attention should be given to this fact. Additionally, it is important to note that the conclusions derived in this paper are only applicable to the nonorthogonal TFCs.

\section{b. Regional Deterministic Prediction System (RDPS)}

It has already been mentioned that the model orography in ECCC's operational 10-km resolution RDPS is based on a cutoff wavelength of $\lambda_{c}=5 \Delta x$ (Separovic et al. 2019). The small-domain LAM test case explored in the preceding subsection has revealed that the issue of spurious amplification of orography-induced waves in the different model-predicted meteorological fields are almost absent for such a filtered orography over the Canadian Rockies for the usual wintertime meteorological conditions. Over the eastern part of North America, the orographic variance is considerably lower than over the west, and thus orography-induced TFC-related numerical noise is generally not an issue for this region. However, careful inspection of some recent RDPS predictions over the eastern United States and Canada has shown that this may not always be the case.

During the second week of February 2020, the jet stream aloft often reached extreme values over the eastern part of North America with the maximum jet speed occasionally exceeding $115 \mathrm{~m} \mathrm{~s}^{-1}$. The jet stream was so strong during this a)

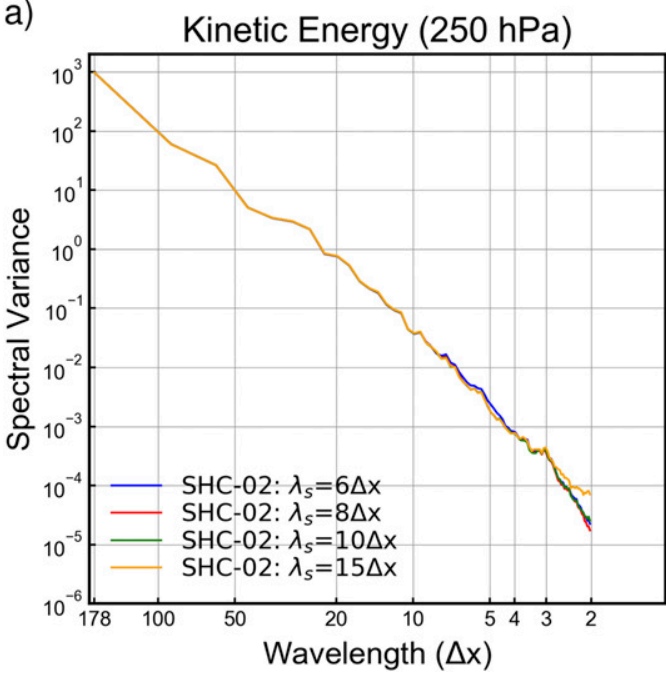

b)

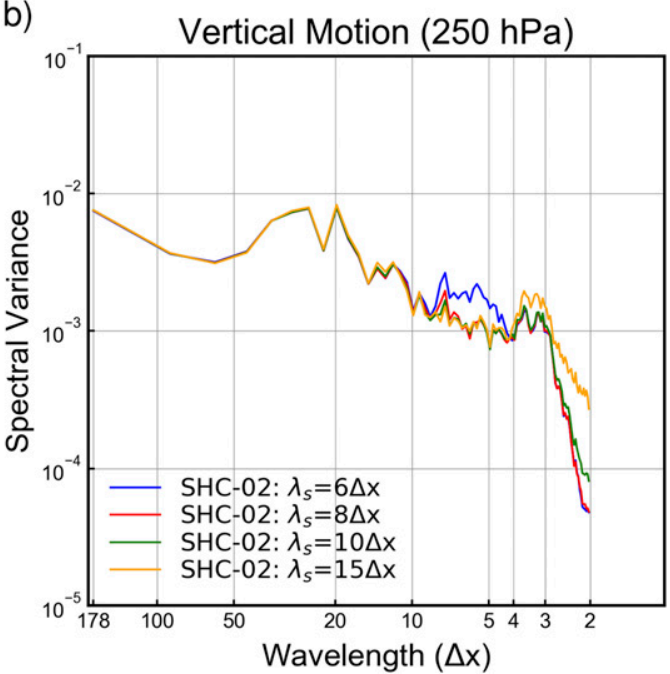

FIG. 10. Spectral variance as a function of wavelengths in terms of grid spacing $(\Delta x=10 \mathrm{~km})$ in (a) kinetic energy and (b) vertical motion at $250 \mathrm{hPa}$ after $24 \mathrm{~h}$ of simulation for the SHC-02 configuration with different values of $\lambda_{s}$ $(6,8,10$, and $15 \Delta x)$ used in determining the large- and small-scale orography. 
a)

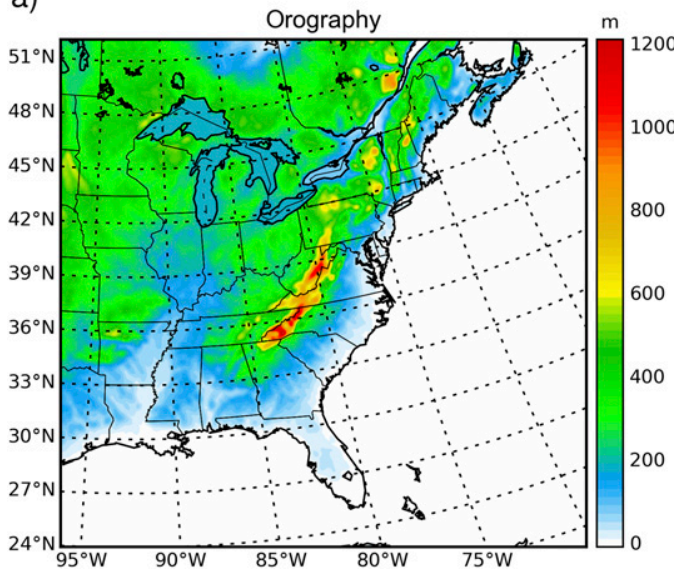

b)

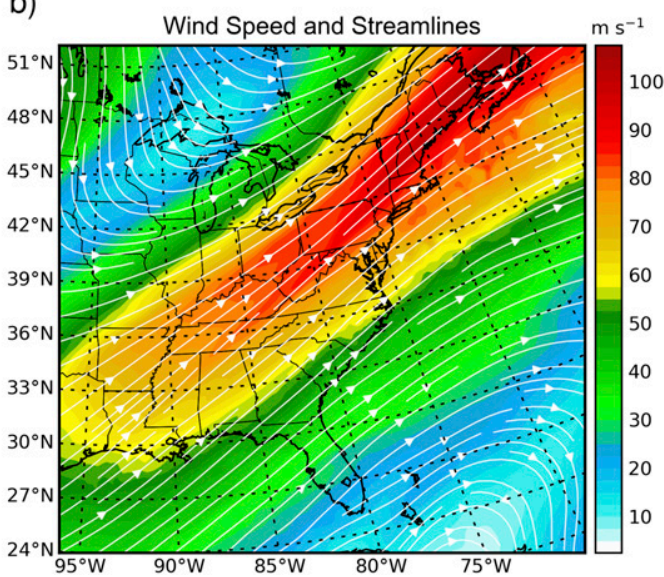

FIG. 11. (a) Orography for the part of ECCC's operational RDPS domain over North America's east coast. Orography is obtained with $\lambda_{c}=5 \Delta x$ in the filter. (b) Map of horizontal wind speed along with the streamlines at $250 \mathrm{hPa}$ after $24 \mathrm{~h}$ of operational RDPS integration initialized at 0000 UTC 13 Feb 2020.

period that multiple cross-Atlantic commercial flights-from New York City, New York, to London, United Kingdom-were able to break the former world record for the fastest subsonic flight. Figure 11a shows part of the operational RDPS domain over the region of interest. The wind speed along with the streamlines at $250 \mathrm{hPa}$ for a forecast lead time of $24 \mathrm{~h}$ for the operational RDPS prediction initialized at 0000 UTC 13 February 2020 are presented in Fig. 11b. The figure shows the upper-air jet stream to be well aligned with the Appalachian Mountain range with the jet intensifying over the northern tip of the mountains.

Overall, some interesting parallels can be drawn between the conditions shown in Fig. 11 to those for the small LAM test case presented earlier. Over the Rockies, a moderately strong jet is found to generate numerically amplified vertical motion in the upper model levels (around $250 \mathrm{hPa}$ ). On the other hand, for this case over the Appalachians, even though the orography amplitudes and variance are less pronounced, the jet speed is substantially stronger. Therefore, the conditions are favorable for generating potentially strong amplification of orographyinduced vertical motions along the direction of the jet, particularly if a sharper resolution orography as in RHC-01 is utilized (i.e., $\lambda_{c}=3 \Delta x$ ). The vertical motion for the 13 February 2020 operational RDPS prediction is shown in Fig. 12a. Interestingly, even with the RHC-02 configuration in the operational RDPS (i.e., $\lambda_{c}=5 \Delta x$ ), the $\omega$ map clearly shows very similar signature of numerically amplified wave structures in the upper-air vertical motion. With the introduction of SHC using $r=(3,15,0,200)$, as can be seen Fig. 12b, this spurious amplification is almost entirely removed. This case is strong evidence that when the conditions are favorable, the TFC-connected spurious amplification of upper-air noise cannot be addressed by simply somewhat increasing the cutoff wavelength in the orography filter. a)

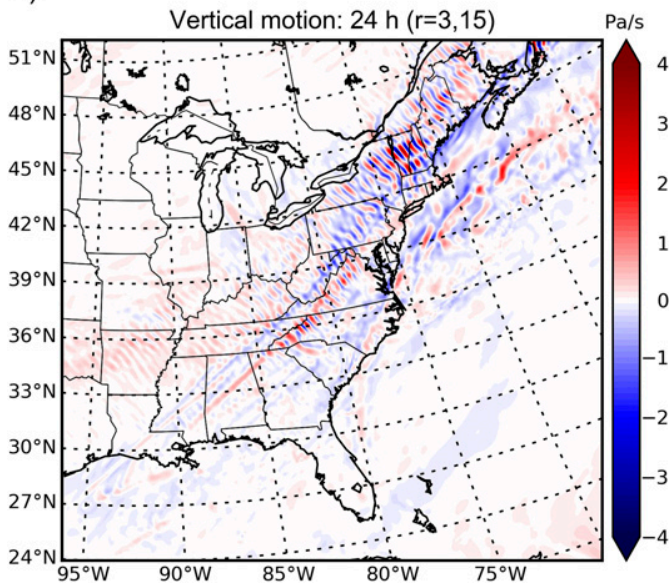

b)

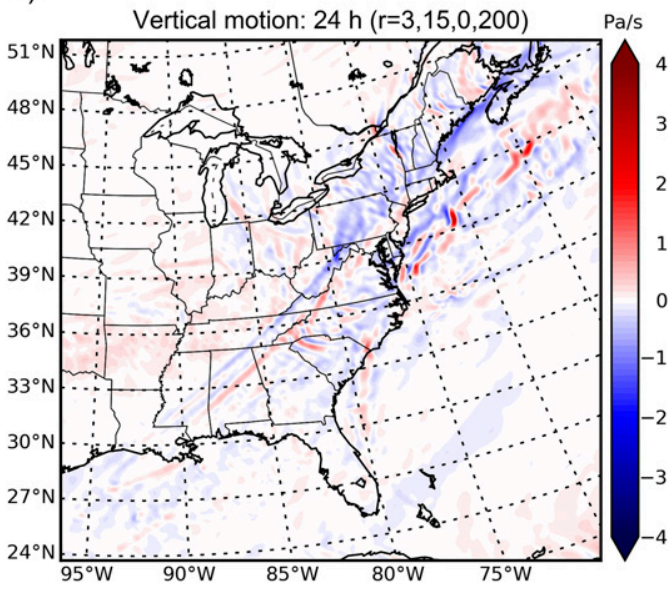

FIG. 12. Vertical motion at $250 \mathrm{hPa}$ after $24 \mathrm{~h}$ of integration for forecast initialized at $0000 \mathrm{UTC} 13 \mathrm{Feb} 2020$ with (a) operational RDPS using $r=(3,15)$ and (b) the SHC approach using $r=(3,15,0,200)$. All other conditions are as in Fig. 11. 
a)

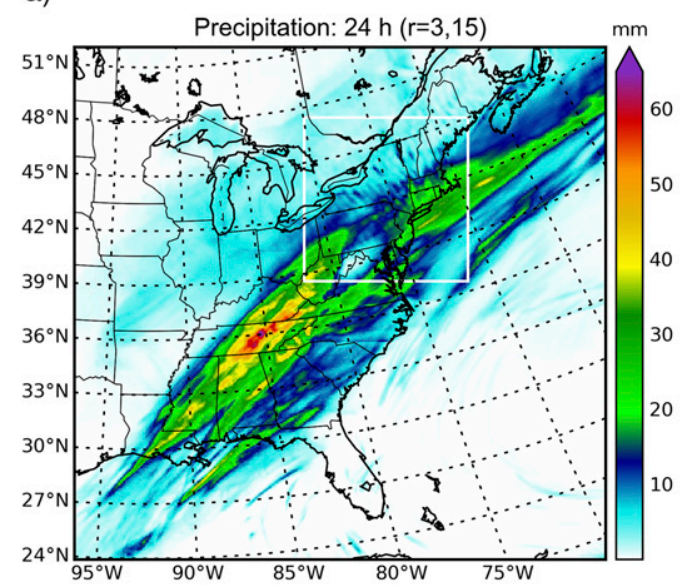

c)

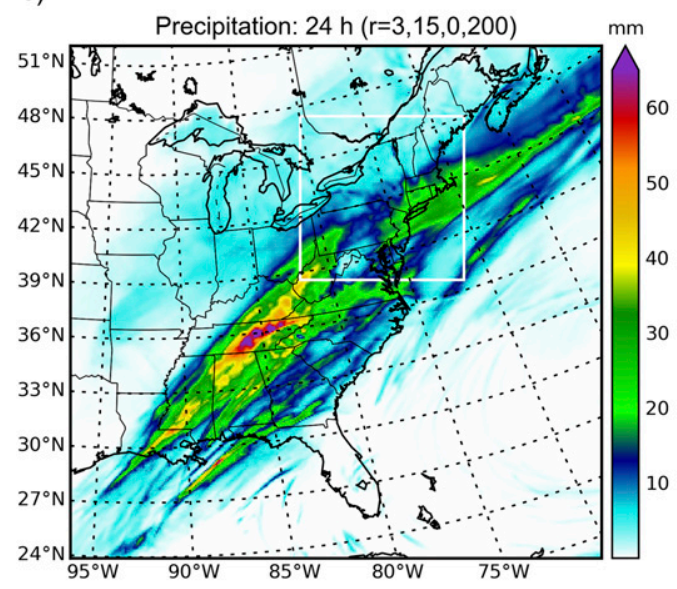

b)

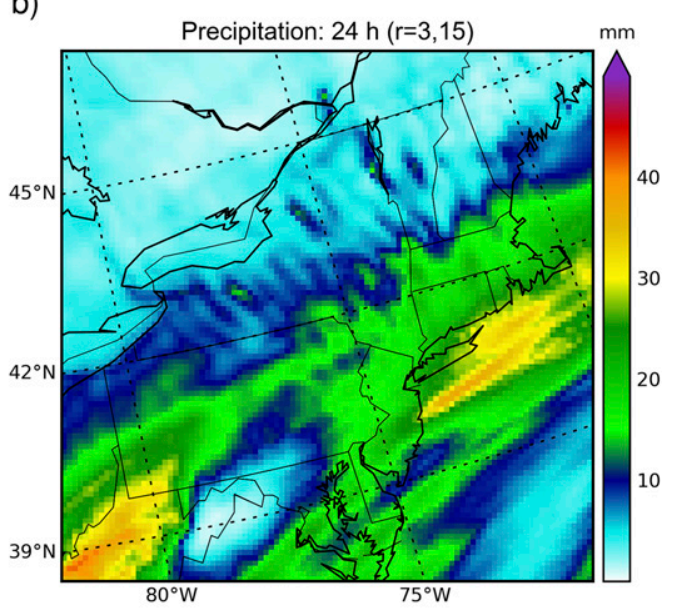

d)

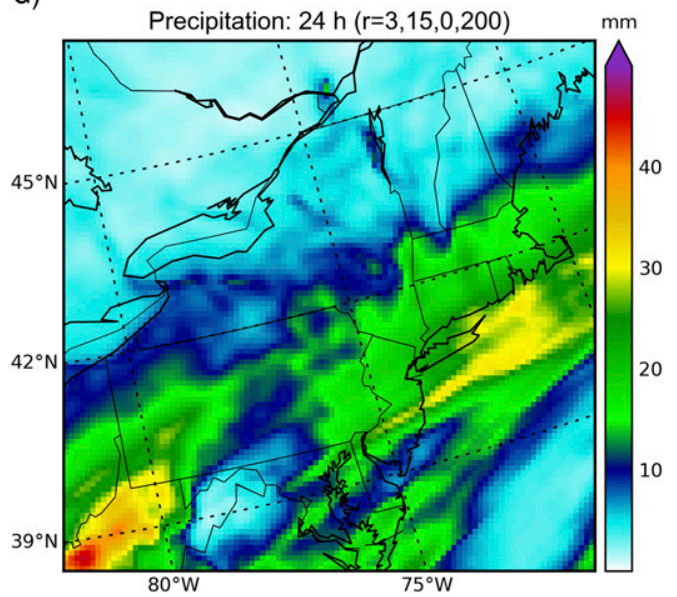

FIG. 13. Distribution of 24-h accumulated precipitation with the operational (top) RDPS and (bottom) SHC. Figures on the right column show the expanded view of the area identified by the white rectangular boxes on the figures within the left column. Coordinates of the four corners of the smaller domain on the right column (clockwise from the bottom-left corner) are $38.598^{\circ} \mathrm{N}, 82.074^{\circ} \mathrm{W} ; 47.462^{\circ} \mathrm{N}, 79.935^{\circ} \mathrm{W} ; 45.009^{\circ} \mathrm{N}, 67.606^{\circ} \mathrm{W}$; and $36.543^{\circ} \mathrm{N}$, $71.649^{\circ} \mathrm{W}$.

Figure 13 presents the 24-h quantitative precipitation forecast (QPF) for this case. Around the northern Appalachians Fig. 13a shows the signature of the spurious vertical motion amplification, particularly within the region bounded by the white square. An enlarged view of this is shown in Fig. 13b. The bands of the wave structures seen in the precipitation field, although of small amplitude, can lead to a reduced confidence in the QPF guidance. The SHC approach, as can be seen in Figs. $13 \mathrm{c}$ and $13 \mathrm{~d}$, efficiently removes these orography-induced wave structures in the precipitation field. Therefore, in addition to improving the upper-air vertical motion and kinetic energy, the SHC approach can be advantageous for improving meteorologically important fields like precipitation from orography-induced TFC-related noise.

\section{c. Global Deterministic Prediction System (GDPS)}

ECCC's current system for global prediction, referred to as the GDPS, became operational on 3 July 2019 (McTaggart-
Cowan et al. 2019). It is based on a horizontal grid resolution of $15 \mathrm{~km}$ and utilizes a sharp-resolution orography with a cutoff length scale of $\lambda_{c}=3 \Delta x$. As a result, the spurious amplification of upper-air vertical motion over complex terrain and the associated effects on other meteorological fields can be occasionally encountered by this system. The GDPS, therefore, provides an excellent opportunity to test how the implementation of SHC would affect the objective forecast scores. To this end, a series of 5-day GDPS forecasts were launched with both the operational (RHC-01) and SHC configurations - covering both winter and summer periods in the Northern Hemisphere (NH). Each seasonal period is represented by 44 cases of consecutive forecasts with initializations that are apart by $36 \mathrm{~h}$ - the first summer case starting at 1200 UTC 17 June 2016, whereas the first winter case starting at 0000 UTC 16 December 2016.

Comparisons between the RHC-01 and SHC-02 configurations for kinetic energy and vertical motion in the spectral space are presented in Fig. 14. Results are shown for both 
a)

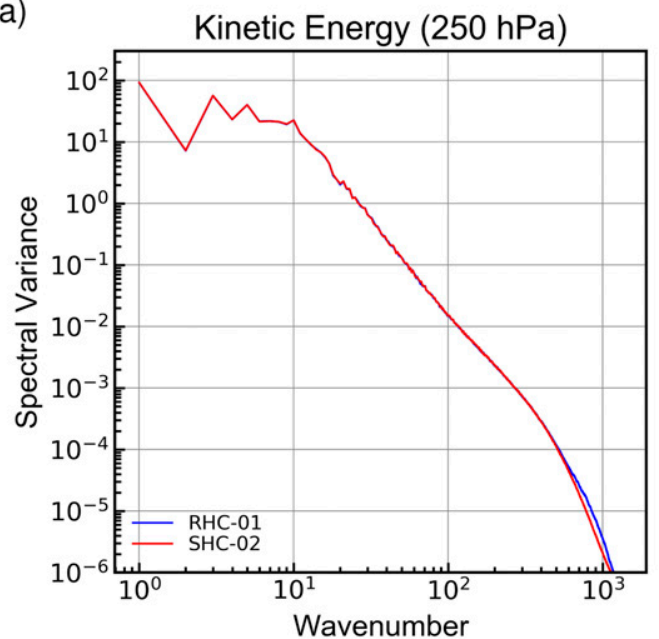

c)

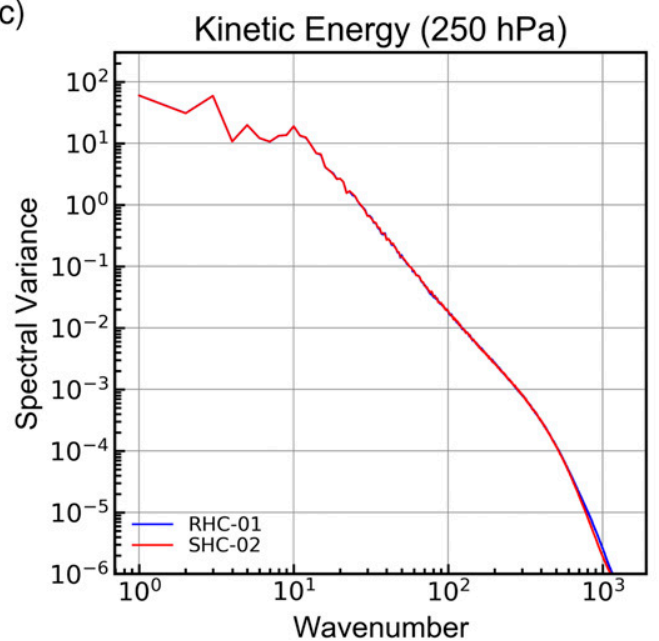

b)

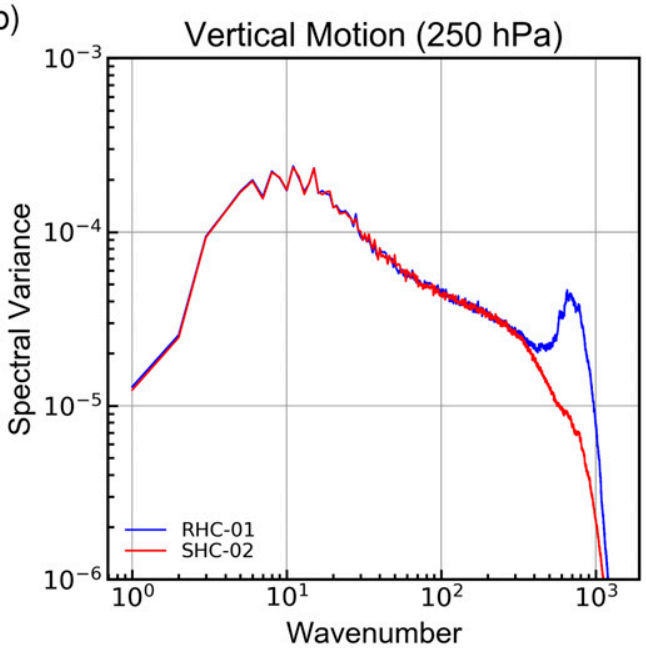

d)

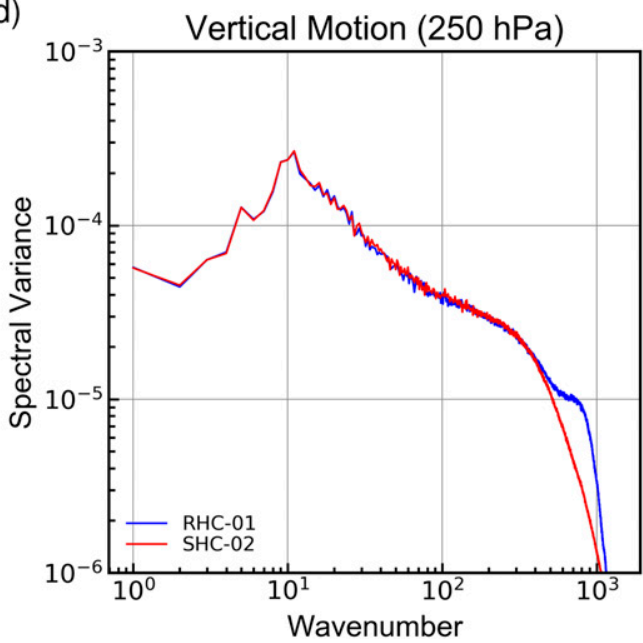

FIG. 14. Global spectra of kinetic energy and vertical motion predicted by the GDPS with RHC-01 (blue, operational) and SHC-02 (red) configurations. Results are obtained by averaging 10 cases of (top) winter and (bottom) summer for the Northern Hemisphere.

$\mathrm{NH}$ winter and summer periods. Over the GDPS domain, the model encounters complex mountain ranges like the Himalayas. Therefore, compared to the RDPS, the SHC configuration in GDPS has to account for orography with significantly steeper slopes and larger variance. With this in mind, the SHC-02 configuration - which allows for some relaxation in the decay of large-scale orography contributions to the model levels while maintaining similar smoothing of the small-scale orography imprints for the upper air-is deemed to be a more prudent choice. The results show some increase in spectral variance at the smallest scales (largest wavenumbers) with the RHC-01 configuration, which is more remarkable for the vertical motion. These finescale variance bumps with RHC-01 are evidently related to the orography imprints in the model vertical coordinate surfaces, and the issue is addressed quite efficiently through the introduction of SHC. It is important to note that the difference in spectra between the two TFC approaches is only visible for spherical wavenumbers larger than 300 , implying that the small-scale spurious variance increase does not affect the synoptic and planetary scales. The variance bump with RHC-01 is much less prominent during the $\mathrm{NH}$ summer period-particularly for the kinetic energy. This is primarily due to the fact that — compared to $\mathrm{NH}$ - the land surface area in the Southern Hemisphere ( $\mathrm{SH})$ is considerably smaller and hence orographic variance is less prominent around the $\mathrm{SH}$ westerly jet.

Figure 15 shows the objective upper-air scores for the winter period that are obtained by comparing the model predictions against observations from radiosondes. The results are presented as vertical profiles of bias and standard deviation of error (SDE) for the average of the 44 cases for this period from the RHC-01 (in blue) and the SHC-02 (in red) configurations. In these error profiles, the appearance of a diamond symbol of a given color (blue or red) at any pressure level indicates that the prediction from the corresponding model configuration delivers statistically significant (with a confidence level above $90 \%$ ) accuracy improvement over the other configuration at 
North America
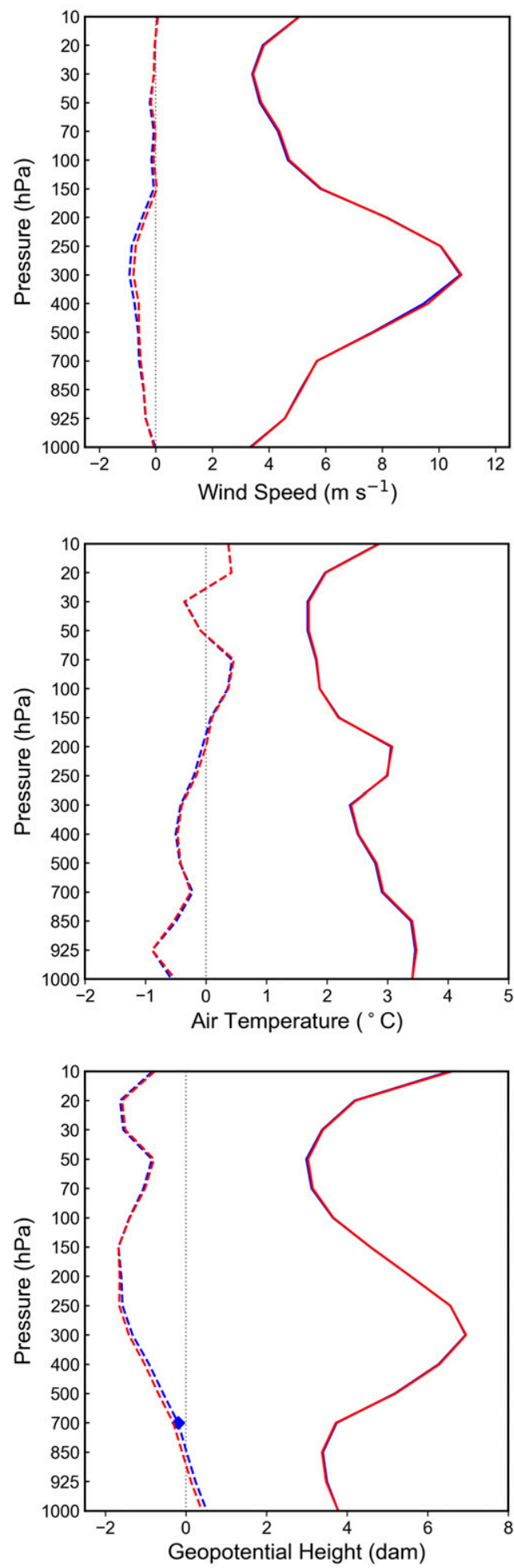

Global
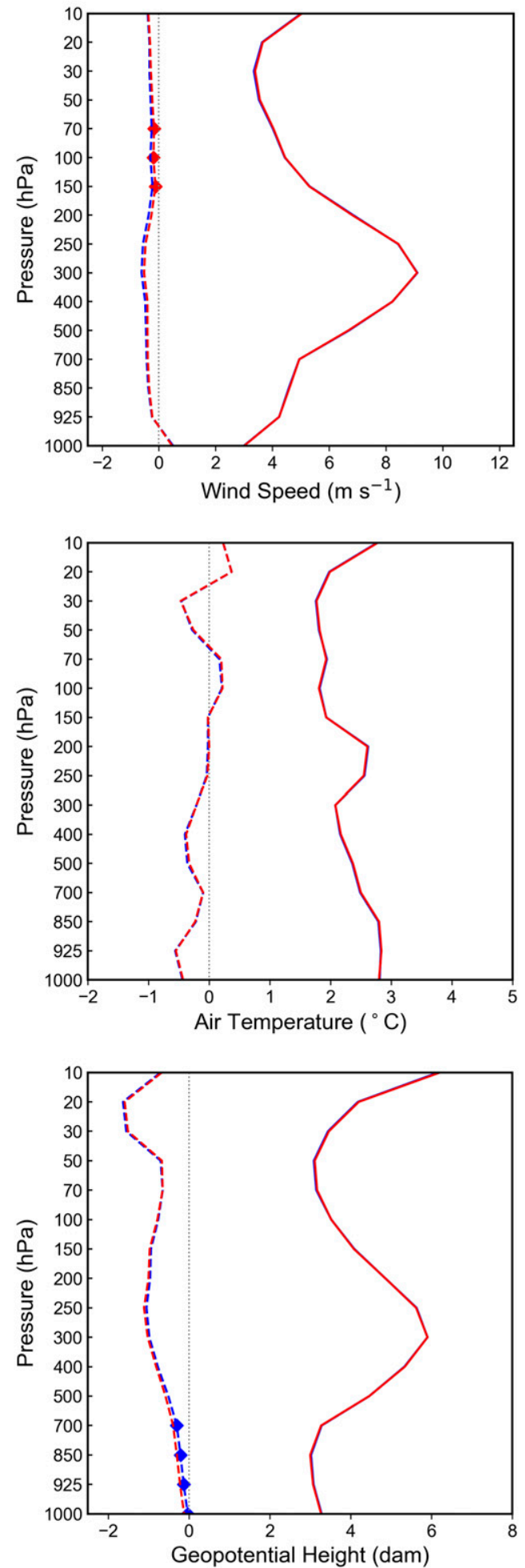

FIG. 15. Upper-air scores for (top) wind speed, (middle) temperature, and (bottom) geopotential height for 15-km GDPS forecasts with the operational RHC (blue, $r=3,15$ ) and SHC (red, $r=0,10,0,200$ ) configurations. The dashed and solid lines denote bias and standard deviation of error, respectively. The results represent (left) North American and (right) global averages of scores obtained for the $44 \mathrm{NH}$ winter cases with a forecast lead time of $120 \mathrm{~h}$. When a model configuration leads to statistically significant improvement in the bias or SDE over the other configuration at a pressure level, the corresponding profile is appended with a diamond-shaped symbol at that level of the color corresponding to associated model configuration (i.e., blue for RHC-01 and red for SHC-02). 
that pressure level. The statistical significance in the bias and SDE are determined using the $t$ and $F$ test, respectively. The 120-h scores between RHC-01 and SHC-02 are found to be largely equivalent - for both North America and the entire globe. Although some statistically significant differences are present in these scores, the physical significance of those differences is only minor, implying that the impact of $\mathrm{SHC}$ is largely neutral. Similar results were also found for the $\mathrm{NH}$ summer period (not shown). These upper-air scores are based on comparisons against observations that are highly irregular in their spatial distribution with inadequate representation over complex terrain. As a result, this is not surprising that the improvements obtained with the SHC approach are not captured by these scores. However, the fact that the objective scores are largely neutral imply that the SHC approach does not affect the model-predicted large-scales that provides confidence for its future implementation in the operational NWP systems.

\section{Summary}

This paper presents a SLEVE-type hybrid coordinate that has been implemented in ECCC's GEM model. By definition, the new coordinate approach accommodates two separate decaying rates for small- and large-scale orography contributions on the model vertical-coordinate surfaces. Near the jet level, particularly over complex terrain, the presence of small-scale variations in the model levels can lead to large error in advection. This in turn induces generation and spurious amplification of vertical motion at the upper levels. Results presented in this paper demonstrate that, in addition to small-scale error in upper-air kinetic energy and vertical motion, the small-scale orography imprints on the coordinate surfaces can lead to deterioration in meteorologically important fields like precipitation.

As the underlying numerical problem is largely driven by the error in semi-Lagrangian advection, it is possible to address the issue by substantially reducing the model time step. Such a solution is, however, not feasible for operational NWP models. Also, filtering the orography further can help by smoothing the orography imprints at all model levels. With the increasing focus on improving orography representation with high-resolution forecasting, further filtering of orography is not an attractive solution either. Moreover, with the help of a recent RDPS case from February 2020, this paper has shown that when the upper-air conditions are favorable, the model outputs with a regular hybrid coordinate can suffer from orography-induced TFC-related noise even with a more filtered orography and also over terrains involving relatively less steep slope and variance. However, a SLEVE-type coordinate, as presented in this paper, can address this numerical issue by simply decaying the small-scale orography faster with height. Results shown in this paper clearly demonstrate that the upperair spurious amplification of vertical motion is effectively eliminated with the SHC approach. The SHC approach also removes any imprint of noise in the vertical motion on the other meteorological fields (e.g., precipitation) that would otherwise be present with the RHC approach. With the help of a series of GDPS cases, it has been shown that the SHC approach can improve the model-predicted small scales while producing largescale forecasts that are statistically equivalent to ECCC's operational global system that utilizes the RHC approach.

It is worth pointing that the SHC configurations used in this study have been selected for demonstrating the potential improvements that are attainable with the SHC approach. It will, however, require a series of extensive tests to identify the optimal values of the exponents of the vertical decay functions for any future implementation of SHC in ECCC's operational NWP systems.

Acknowledgments. The authors would like to sincerely thank the members of RPN-A for all their thoughtful comments and suggestions during the course of this work.

\section{APPENDIX}

\section{Suitable Range of Values for the Exponent $r$}

Too large of a value of $r$ can lead to intersecting vertical coordinate surfaces near the mountain tops and thereby violate the monotonicity condition that requires $(\partial \ln \pi / \partial \zeta)>0$, which implies that in order to ensure monotonicity,

$$
\frac{\ln \left(p_{\mathrm{ref}} / \pi_{S}\right)}{\ln \left(p_{\mathrm{ref}} / \pi_{T}\right)} \frac{\partial B}{\partial \psi}<1,
$$

is to be satisfied at every model level. It is therefore important to ascertain an idea about the maximum allowable value of $r$ that would not violate the monotonicity requirement.

The first term in Eq. (A1) is always less than 1, as $\pi_{T}<\pi_{S}$. The second term then can be expressed as

$$
\frac{\partial B}{\partial \psi}=r \frac{B}{\psi}
$$

where $B / \psi$ has a maximum value of 1 at the surface [see Eq. (2)]. Therefore, the $(\partial B / \partial \psi)_{\max }=r$ at the surface. The monotonicity condition thus reduces to $r<\left[\ln \left(p_{\text {ref }} / \pi_{T}\right) / \ln \left(p_{\text {ref }} / \pi_{S}\right)\right]$. For example, if $\pi_{T}$ is set to $10 \mathrm{~Pa}$ then over the peak of the Everest, where surface pressure is approximately $0.32 p_{\text {ref }}$, the maximum allowable value of $r$ is around 2.95. However, for the different GEM-based operational NWP systems - owing to the model resolutions and application of orography filters - the minimum value of $\pi_{S}$ is usually in the range of $0.45 p_{\text {ref }}-0.55 p_{\text {ref }}$. Therefore, depending on an NWP system's domain of coverage, spatial resolution and the resolution of the resolved orography, larger values of $r$ (i.e., $r>2.95$ ) are allowable.

\section{REFERENCES}

Elvidge, A. D., and Coauthors, 2019: Uncertainty in the representation of orography in weather and climate models and implications for parameterized drag. J. Adv. Model. Earth Syst., 11, 2567-2585, https://doi.org/10.1029/2019MS001661.

Girard, C., R. Benoit, and M. Desgagné, 2005: Finescale topography and the MC2 dynamics kernel. Mon. Wea. Rev., 133, 14631477, https://doi.org/10.1175/MWR2931.1.

— , and Coauthors, 2014: Staggered vertical discretization of the Canadian Environmental Multiscale (GEM) model using a coordinate of the log-hydrostatic-pressure type. Mon. Wea. Rev., 142, 1183-1196, https://doi.org/10.1175/MWR-D-13-00255.1. 
Husain, S. Z., and C. Girard, 2017: Impact of consistent semiLagrangian trajectory calculations on numerical weather prediction performance. Mon. Wea. Rev., 145, 4127-4150, https://doi.org/10.1175/MWR-D-17-0138.1.

$\longrightarrow,-$, A. Qaddouri, and A. Plante, 2019: A new dynamical core of the Global Environmental Multiscale (GEM) model with a height-based terrain-following vertical coordinate. Mon. Wea. Rev., 147, 2555-2578, https://doi.org/10.1175/MWR-D-18-0438.1.

Klemp, J. B., 2011: A terrrain-following coordinate with smoothed coordinate surfaces. Mon. Wea. Rev., 139, 2163-2169, https:// doi.org/10.1175/MWR-D-10-05046.1.

Lander, J., and B. J. Hoskins, 1997: Determining the effective resolution of advection schemes. Part I: Dispersion analysis. J. Comput. Phys., 278, 485-496, https://doi.org/10.1016/J.JCP.2014.01.043.

Laprise, R., 1992: The Euler equations of motion with hydrostatic pressure as an independent variable. Mon. Wea. Rev., 120, 197-207, https://doi.org/10.1175/1520-0493(1992)120<0197: TEEOMW $>2.0 . \mathrm{CO} ; 2$.

Leuenberger, D., M. Koller, O. Fuhrer, and C. Schär, 2010: A generalization of the SLEVE vertical coordinate. Mon. Wea. Rev., 138, 3683-3689, https://doi.org/10.1175/2010MWR3307.1.

Li, Y., B. Wang, D. Wang, and L. Dong, 2014: An orthogonal terrain-following coordinate and its preliminary tests using 2-D idealized advection experiments. Geosci. Model Dev., 7, 1767-1778, https://doi.org/10.5194/gmd-7-1767-2014.

Mahrer, Y., 1984: An improved numerical approximation of the horizontal gradients in a terrain-following coordinate system. Mon. Wea. Rev., 112, 918-922, https://doi.org/10.1175/15200493(1984)112<0918:AINAOT>2.0.CO;2.

McTaggart-Cowan, R., and Coauthors, 2019: Modernization of atmospheric physics parameterization in Canadian NWP. J. Adv. Model. Earth Syst., 11, 3593-3635, https://doi.org/ 10.1029/2019MS001781.

Park, S.-H., J. B. Klemp, and J.-H. Kim, 2019: Hybrid mass coordinate in WRF-ARW and its impact on upper-level turbulence forecasting. Mon. Wea. Rev., 147, 971-985, https:// doi.org/10.1175/MWR-D-18-0334.1.
Qaddouri, A., and V. Lee, 2011: The Canadian Global Environmental Multiscale model on the Yin-Yang grid system. Quart. J. Roy. Meteor. Soc., 137, 1913-1926, https://doi.org/10.1002/qj.873.

Schär, C., D. Leuenberger, O. Fuhrer, D. Lüthi, and C. Girard, 2002: A new terrain-following vertical coordinate formulation for atmospheric prediction models. Mon. Wea. Rev., 130, 2459-2480, https://doi.org/10.1175/1520-0493(2002)130<2459: ANTFVC $>2.0 . \mathrm{CO} ; 2$.

Separovic, L., and Coauthors, 2019: Changes to the Regional Deterministic Prediction System (RDPS) from version 6.0.0 to version 7.0.0. Canadian Meteorological Centre Tech. Note, Environment and Climate Change Canada, 45 pp., https:// collaboration.cmc.ec.gc.ca/cmc/cmoi/product_guide/docs/ tech_notes/technote_rdps-700_e.pdf.

Simmons, A. J., and D. M. Burridge, 1981: An energy and angularmomentum conserving finite-difference scheme and hybrid vertical coordinates. Mon. Wea. Rev., 109, 758-766, https://doi.org/ 10.1175/1520-0493(1981)109<0758:AEAAMC >2.0.CO;2.

Skamarock, W. C., 2004: Evaluating mesoscale NWP models using kinetic energy spectra. Mon. Wea. Rev., 132, 3019-3032, https://doi.org/10.1175/MWR2830.1.

Vionnet, V., S. Bélair, C. Girard, and A. Plante, 2015: Wintertime subkilometer numerical forecasts of near-surface variables in the Canadian Rocky Mountains. Mon. Wea. Rev., 143, 666686, https://doi.org/10.1175/MWR-D-14-00128.1.

Wood, N., and Coauthors, 2014: An inherently mass-conserving semi-implicit semi-Lagrangian discretization of the deepatmosphere global non-hydrostatic equations. Quart. J. Roy. Meteor. Soc., 140, 1505-1520, https://doi.org/10.1002/qj.2235.

Zadra, A., 2018: Notes on the new low-pass filter for the orography field. Internal Rep. RPN-A, Meteorological Research Division, Environment and Climate Change Canada, 11 pp., http://collaboration.cmc.ec.gc.ca/science/rpn/drag_project/ documents/topo_lowpass_filter.pdf.

Zängl, G., 2012: Extending the numerical stability limit of terrainfollowing coordinate models over steep slopes. Mon. Wea. Rev., 140, 3722-3733, https://doi.org/10.1175/MWR-D-12-00049.1. 